\title{
EL PAPEL DE LA UE COMO GARANTE DEL ESTADO DE DERECHO EN RELACIÓN CON EL SISTEMA DE SOLUCIÓN DE DIFERENCIAS DE LA OMC'
}

\author{
CARMEN LÓPEZ-JURADO ROMERO DE LA CRUZ² \\ lopezju@ugr.es
}

\begin{abstract}
Cómo citar/Citation
López-Jurado Romero de la Cruz, C. (2021).

El papel de la UE como garante del estado de derecho en relación con el sistema de solución de diferencias de la OMC. Revista de Derecho Comunitario Europeo, 70, 869-896.

doi: https://doi.org/10.18042/cepc/rdce.70.02
\end{abstract}

\section{Resumen}

El sistema de solución de controversias de la Organización Mundial del Comercio es un componente esencial de la rule of law del comercio internacional. Sin embargo, debido al bloqueo por parte de Estados Unidos del nombramiento de nuevos miembros del Órgano de Apelación, este mecanismo está en crisis. El objetivo de este trabajo es investigar las propuestas de la Unión Europea dirigidas a desbloquear la situación de crisis. Ello exige determinar con carácter previo tanto el contexto económico en el que se ha gestado esta crisis como las críticas estadounidenses que subyacen a su decisión de bloquear el funcionamiento del Órgano de Apelación.

\section{Palabras clave}

Organización Mundial del Comercio; Unión Europea; Estado de Derecho; sistema de solución de diferencias de la OMC; informe Lighthizer; informe Walker;

1 Este trabajo se ha realizado en el marco del Proyecto de investigación del Ministerio de Ciencia, Innovación y Universidades titulado «La acción exterior de la Unión Europea y el Estado de Derecho» (RTI2018-101041-B-100), del que son investigadores principales la Profa. Dra. Carmela Pérez Bernárdez y el Prof. Dr. Luis Miguel Hinojosa Martínez.

2 Profesora titular de Derecho Internacional Público, Universidad de Granada. 
Acuerdo Multilateral de Arbitraje de Apelación del artículo 25 del Entendimiento sobre Solución de Diferencias; reforma de la política comercial de la UE.

\title{
THE ROLE OF THE EU AS GUARANTOR OF THE RULE OF LAW IN RELATION TO THE WTO'S DISPUTE SETTLEMENT SYSTEM
}

\begin{abstract}
The WTO dispute settlement system is an essential component of the rule of law in international trade. However, due to the United States blocking the appointment of new Appellate Body members this mechanism is in crisis. The aim of this paper is to investigate the European Union proposals aimed at unblocking the crisis situation. This requires first determining both the economic context in which this crisis has arisen and the United States criticism that underlies its decision to block the functioning of the Appellate Body.
\end{abstract}

\section{Keywords}

World Trade Organization; European Union; Rule of Law; WTO Dispute Settlement Crisis; Lighthizer Report; Walker Report; Multi-Party Interim Appeal Arbitration Arrangement pursuant Article 25 of the Dispute Settlement Understanding; EU Trade Policy Reform.

\section{LE RÔLE DE L'UE EN TANT QUE GARANT DE L'ÉTAT DE DROIT DANS LE CADRE DU SYSTÈME DE RÈGLEMENT DES DIFFÉRENDS DE L'OMC}

\section{Résumé}

Le système de règlement des différends de l'OMC est une composante essentielle de l'état de droit dans le commerce international. Toutefois, en raison du blocage par les États-Unis de la nomination des nouveaux membres de l'Organe d'appel, ce mécanisme est en crise. L'objectif de ce document est d'examiner les propositions de l'Union Européenne visant à débloquer la situation de crise. Pour ce faire, il faut d'abord identifier à la fois le contexte économique dans lequel cette crise s'est produite et la critique américaine qui sous-tend sa décision de bloquer le fonctionnement de l'Organe d'appel.

\section{Mots clés}

Organisation mondiale du commerce; Union Européenne; État de droit; Système de règlement des différends de l'OMC; Rapport Lighthizer; Rapport Walker; Accord multilatéral sur l'arbitrage; Article 25 du Mémorandum d'accord sur le règlement des différends; Réforme de la politique commerciale de l'Union Européenne. 


\section{SUMARIO}

I. INTRODUCCIÓN. II. UN CONTEXTO GLOBAL INTERNACIONAL DE GUERRA COMERCIAL. III. EL BLOQUEO DEL ÓRGANO DE APELACIÓN. IV. EL INFORME LIGHTHIZER: 1. Críticas de carácter procedimental. 2. Críticas de carácter sistémico. 3. Críticas de tipo sustantivo. V. PROPUESTAS DE DESBLOQUEO DEL SISTEMA DE SOLUCIÓN DE DIFERENCIAS DE LA OMC: 1. La iniciativa de la UE para desbloquear la parálisis del SSD. 2. El proceso informal sobre cuestiones relacionadas con el funcionamiento del Órgano de Apelación. 3. El Acuerdo Multilateral de Arbitraje de Apelación. 4. La aplicación de contramedidas unilaterales por parte de la UE en relación con países no participantes en el MPIA. 5. El SSD a la luz de la reforma de la Política comercial de la UE. VI. REFLEXIONES FINALES. BIBLIOGRAFía.

\section{INTRODUCCIÓN}

El Sistema de Solución de Diferencias (SSD, en adelante) de la Organización Mundial del Comercio (OMC, en lo sucesivo) es un mecanismo diseñado para asegurar el cumplimiento del derecho de la OMC, lo que contribuye a aportar seguridad y previsibilidad al sistema de normas integradas en esta importante organización internacional. Las modificaciones efectuadas durante la ronda de Uruguay, especialmente la creación de un Órgano permanente de Apelación (OA, en lo sucesivo), o la incorporación de la regla del consenso negativo en el Órgano de Solución de Diferencias (OSD, en adelante) para la adopción de los informes emitidos en las diferentes instancias de solución de diferencias, contribuyeron a configurarlo como un mecanismo de solución de diferencias absolutamente innovador.

Regulado en el Entendimiento sobre Solución de Diferencias (ESD), el procedimiento de solución de diferencias se sustancia, en primera instancia, ante los denominados grupos especiales, más conocidos en la jerga especializada como panels, cuyos informes pueden ser recurridos ante el OA. Los informes emitidos tanto por los grupos especiales como por el OA han de ser finalmente adoptados por el OSD para devenir obligatorios. Muestra de la ausencia de jerarquía de los modos de solución de diferencias en el marco de la OMC (Carreau y Juillard, 2017: 131), el ESD también contempla la posibilidad de que las partes en una diferencia comercial acudan paralelamente a 
otros medios de solución de diferencias, que pueden ser tanto de carácter diplomático (buenos oficios, mediación y conciliación) como de carácter jurisdiccional (arbitraje).

No cabe duda de la importancia que tiene el SSD de la OMC en la progresiva articulación del Estado de Derecho en la esfera comercial internacional. Se trata de una noción esta en la que subyacen conceptos tales como el de la separación de poderes, la independencia del poder judicial o la igualdad ante el derecho. En el caso del SSD de la OMC, cabe apreciar estos elementos en: a) la existencia de un órgano de naturaleza quasi jurisdiccional como el OA, del que Weiler ha subrayado que es un tribunal en todo salvo en el nombre (Weiler 2001: 201); b) la creación de un procedimiento reglado, al que tienen acceso todos los miembros de la OMC, cuyo último fin es el cumplimiento de los acuerdos abarcados; y, c) el establecimiento de mecanismos de control específicos para exigir el cumplimiento de los informes adoptados.

De este modo, no resulta extraño que la doctrina especializada afirme que instituciones como el SSD de la OMC contribuyen significativamente a afianzar un verdadero Estado de Derecho en el ámbito internacional (Hillman, 2010: 269); o que se haya llegado a considerar al SSD como un "componente crítico» de la rule of law del comercio internacional (McDougal, 2021: 197), incluso "a glorious experiment with the rule of law in international relations» (Van den Bossche, citado por Pauwelyn, 2019: 298). En esta línea, otros autores consideran que el OA ha contribuido de modo decisivo a reforzar la rule of law del comercio internacional (Lacarte-Muro y Ganesan, citados por Hoekman y Mavroidis, 2019b: 14). Por su parte, Petersmann, ha llegado a identificar la rule of law del sistema económico internacional en el ESD (Petersmann, 2018a: 112 y 121).

Sin embargo, a pesar de su importancia objetiva tanto en términos generales como en lo que respecta a su contribución a la consecución del Estado de Derecho en la esfera internacional, en los últimos años este SSD está en crisis debido a la decidida política estadounidense de bloquear el nombramiento de nuevos miembros del OA. La situación así creada está impidiendo resolver, en esa segunda instancia, los recursos presentados contra los informes de los grupos especiales, abocando a la OMC a una situación de crisis sin precedentes.

Ello ha llevado a la Unión Europea (UE, en adelante), organización internacional frecuentemente calificada como potencia normativa, a intentar buscar soluciones de carácter multilateral que permitan superar, siquiera provisionalmente, el colapso del sistema comercial internacional. En este sentido, cabe recordar que la UE se ha erigido como garante del Estado de Derecho tanto en el ámbito interno, es decir, en relación con sus propios Estados miembros (y ahí están tanto el art. 7 del TUE como el art. 19 del TUE, en relación con 
el art. 258 del TFUE), como en el ámbito de las relaciones exteriores, esto es, en relación con terceros, función esta última que le asigna el art. 21 del TUE 3 , y que es, a su vez, un objetivo de la UE (art. 3. 5 del TUE). De este modo, «el Estado de Derecho se ha convertido en un elemento esencial de la acción exterior de la UE» (Liñán, 2018: 61, Roldán, 2018: 231), lo que presenta diferentes manifestaciones. Así, es un eje central de la Nueva Estrategia Global para la Política Exterior y de Seguridad de la UE4; también lo es de la Estrategia para la ampliación de la UE ${ }^{5}$ (López-Jurado, 2018: 425) y, ahora, como vemos, en el ámbito de las relaciones comerciales internacionales.

El objetivo de este trabajo será investigar el papel de la UE como garante del Estado de Derecho en relación con el SSD de la OMC, lo que exigirá determinar con carácter previo tanto el contexto económico en el que se ha gestado esta crisis como las críticas estadounidenses —al SSD de la OMCque subyacen en su decisión final de bloquear el funcionamiento del OA.

\section{UN CONTEXTO GLOBAL INTERNACIONAL DE GUERRA COMERCIAL}

Es importante situar la especial coyuntura que atraviesa el SSD de la OMC en el momento actual en el contexto más amplio de guerra comercial internacional. Ya en 2002 Estados Unidos (EE. UU., en lo sucesivo) decidió aplicar de modo unilateral aranceles a los productos del acero, siendo denunciado ante la OMC por la UE, lo que dio lugar a varios informes desfavorables para EE. UU. tanto del grupo especial como del $\mathrm{OA}^{6}$.

La llegada al poder de Donald Trump en 2017, con su lema nacionalista America first, llevó consigo una escalada del unilateralismo en la política comercial estadounidense, lo que derivó en la adopción de otras medidas de carácter proteccionistas al acero y al aluminio con base jurídica en la sección

3 Según el art. 21.1: «La acción de la Unión en la escena internacional se basará en los principios que han inspirado su creación, desarrollo y ampliación y que pretende fomentar en el resto del mundo: la democracia, el Estado de Derecho, la universalidad e indivisibilidad de los derechos humanos y de las libertades fundamentales, el respeto de la dignidad humana, los principios de igualdad y solidaridad y el respeto de los principios de la Carta de las Naciones Unidas y del Derecho Internacional».

4 El documento, que lleva como título Una visión común, una estrategia conjunta, una Europa más fuerte, está disponible en: https://bit.ly/2ZztvS1.

5 Comisión Europea, Comunicación sobre la política de ampliación de la UE en 2016, COM (2016) 715 final, de 9 de noviembre de 2016, pp. 3-4.

6 OMC, Estados Unidos - Medidas de salvaguardia definitivas sobre las importaciones de determinados productos de acero, WT/DS248 y siguientes. 
232 de la Trade Expansion Act de 1962, una ley que otorga poderes al presidente de los EE. UU. para actuar en el terreno comercial cuando considera que determinadas importaciones amenazan la seguridad nacional. De este modo, a los efectos de la aplicación de la sección 232, el término «seguridad nacional» se equiparaba a "seguridad económica» (McDougal, 2018: 869). Desde el punto de vista jurídico, ello implicó que el debate pasaba de estar centrado en la licitud de las medidas (sobre la base del Acuerdo sobre salvaguardias) a su adecuación a la excepción relativa a la seguridad nacional del art. XXI del GATT de 1994 (Magi, 2020: 34), con lo que ello entrañaba de discrecionalidad; y propició, a su vez, la conclusión de acuerdos bilaterales entre EE. UU. y determinados países (Australia, Argentina, Brasil, Corea del Sur) cuyas exportaciones a EE. UU. de tales productos quedaban exentas de las «medidas de seguridad nacional» así adoptadas.

Las relaciones con el resto de miembros de la OMC se deterioraron progresivamente dando lugar a una abierta guerra comercial que presentaba, en su núcleo, una disputa entre los grandes del comercio internacional (EE. UU., China, la UE), y a la que se fueron añadiendo extensiones en función del haz de relaciones bilaterales que tenía cada uno de ellos. La guerra comercial acabó afectando a un listado cada vez mayor de productos, mostrando al mundo la impotencia del sistema multilateral en su formato actual para gestionar las relaciones de fuerza existentes en el ámbito comercial internacional (Hamann, 2018: 696).

En las relaciones entre EE. UU. y la UE la crisis ha vivido su punto más álgido con la aplicación de contramedidas cruzadas en la controversia paralela entre EE. UU. y la UE relativa a las subvenciones a la industria aeronáutica (Airbus/Boeing). El cambio de Administración estadounidense, tras la llegada al poder de Biden, ha supuesto un acercamiento entre las posiciones de ambas partes, que se ha materializado en la suspensión, durante cuatro meses, de los aranceles establecidos a ambos lados del Atlántico, beneficiando a numerosas empresas y restaurando las relaciones entre EE. UU. y la UE. En una Declaración conjunta de 3 de marzo de 2021 se señala que ello permitirá a la UE y a los EE. UU. aliviar la carga a sus industrias y trabajadores y centrar los esfuerzos en la resolución sus largas diferencias en el marco de la OMC.

Una segunda vertiente de esta guerra comercial internacional tiene que ver con el tratamiento que la OMC otorga a China. En relación con esta cuestión, las críticas estadounidenses son compartidas por la UE. Recordemos que la adhesión de China a la OMC se produjo en 2001 y que este país ha pasado, tan solo en un plazo de quince años, de ser considerado como una economía planificada (non-market economy) a pretender ser incluido en la categoría de las economías de mercado. La situación anómala de la que disfruta China en la $\mathrm{OMC}$ le permite beneficiarse de un trato discriminatorio preferencial respecto 
al resto de los miembros de la OMC con sistemas económicos neoliberales (Bhala et al., 2020: 55). EE. UU. también cuestiona la endémica vulneración por parte de China del Acuerdo sobre los Derechos a la Propiedad Intelectual relacionados con el Comercio (ADPIC), la existencia de obstáculos técnicos al comercio a la importación de mercancías, las subvenciones que otorga a sus empresas —en realidad conglomerados de carácter público-privado-, el disponer de un acceso ilimitado al crédito (Féas y Steinberg, 2020: 78), la manipulación del tipo de cambio de su moneda, o sus prácticas de dumping social y ambiental, prácticas estas últimas que no pueden ser adecuadamente cuestionadas bajo las vigentes normas substantivas de la OMC. Esta argumentación ha sido utilizada también por la Administración Trump para autorizar al representante de comercio de los EE. UU. a llevar a cabo una investigación, basada en la sección 302(b) de la Trade Act [19 U.S.C. 2412 (b)] con objeto de determinar si los actos, políticas y prácticas de China relacionadas con la transferencia de tecnología, propiedad intelectual e innovación son discriminatorios y restringen el comercio estadounidense. McDougal considera razonable sospechar que EE. UU., al bloquear el OA, está intentando frenar la consolidación de la ventaja comparativa que el actual sistema normativo de la OMC concede a China (McDougal, 2018: 872), especialmente teniendo en cuenta la forma en que este Derecho es interpretado por el OA.

\section{EL BLOQUEO DEL ÓRGANO DE APELACIÓN}

La táctica estadounidense para erosionar el SSD de la OMC ha evolucionado con el paso del tiempo. Inicialmente consistió en el bloqueo de la renovación del mandato de determinados miembros del OA. Esta suerte de bloqueo se hizo notar durante la presidencia de Barack Obama con la falta de renovación de los mandatos de aquellos miembros del OA que habían participado en apelaciones que fueron consideradas desfavorables para los EE. UU. (Kuijper, 2017: 3). Así ocurrió con la estadounidense Hillman y con el surcoreano Chang, dando lugar, en este último caso, a acalorados debates en el seno del OSD de la $\mathrm{OMC}^{7}$. La doctrina especializada vio en esta política estadounidense el reflejo de una politización en los nombramientos de los miembros del OA poco acorde con el papel de independencia que les corresponde (Hufbahuer, 2011). La táctica estadounidense dio lugar también a un

7 WT/DSB/M/379, de 29.8.2016, en particular el apdo. 6. Véase, en relación con este último caso, «United States Blocks Reappointment of WTO Appellate Body Member», $A J I L$, 2016, pp. 573-579. 
debate en el que diversos miembros de la OMC subrayaron el temor a que, de este modo, se pudiera comprometer la integridad e imparcialidad del OA, al presionar a sus miembros para que resolvieran "de una determinada manera» (AJIL, 2016: 577). No resulta extraño, en este sentido, que los restantes miembros del OA llegaran a dirigir una carta al director general de la OMC mostrando su disconformidad respecto a la actuación estadounidense (Kanth, 2016).

Es importante señalar a este respecto que los miembros del OA pueden ser renovados una vez por un periodo de cuatro años, y que la designación de los miembros del OA se efectúa, en el seno del OSD, por consenso (en este caso positivo), lo que este exige que ningún miembro presente en la reunión del OSD en que se adopte la decisión se oponga formalmente a ella (art. 2. 4 del ESD). Cabe subrayar, además, que la forma de designación de los miembros del OA contrasta con la seguida en relación con los grupos especiales, en los que, a falta de acuerdo entre las partes en la diferencia respecto a su nombramiento, es el director general de la OMC quien los nombra, incluso con la oposición de las partes, de modo que, en relación con estos, no cabe el ejercicio de un derecho de veto (Malacrida y Marceau, 2018: 27).

El mal presagio que auguraba la táctica estadounidense respecto a la falta de renovación de los mandatos de determinados miembros del OA se hizo realidad cuando la Administración Trump decidió dar un salto cualitativo en su agresiva política comercial adoptando como estrategia el bloqueo del nombramiento de nuevos miembros del OA. Ello trajo como consecuencia el que, desde el 11 de diciembre de 2019, de los siete miembros que debía haber en este órgano - tres, como mínimo, para resolver una apelación-, solo quedaba uno (cuyo mandato además estaba llamado a expirar el 30 de noviembre de 2020), lo que hacía imposible resolver en esa instancia las demandas presentadas. Por consiguiente, desde entonces, cualquier miembro de la OMC puede evitar que se adopte una resolución vinculante en el marco del SSD de la OMC simplemente presentando un recurso ante el OA contra un informe de un grupo especial que le sea desfavorable. Dado que el OA no puede ocuparse de nuevos recursos (porque no tiene el número mínimo de miembros para hacerlo), el litigio queda en un vacío legal y no se puede resolver (lo que se denomina un recurso «nulo de pleno derecho»).

El OA subrayó en su informe anual correspondiente a 2018 la crisis institucional a que conducía la táctica unilateral estadounidense, precisando que el número reducido de sus miembros, que era entonces de tres, menoscababa la colegialidad de las deliberaciones, incrementaba los retrasos en los procedimientos de apelación y cuestionaba su propia legitimidad. La Administración estadounidense se limitó a subrayar que EE. UU. perdía casi todas las disputas comerciales, a pesar del hecho constatado por la doctrina de que EE. UU. 
ha ganado el $75 \%$ de sus demandas ante el SSD de la OMC (Petersmann, 2018b: 2). Se trata, en realidad, de una "verdad a medias» ya que mientras que EE. UU. ha ganado en términos generales los casos resueltos por el SSD, no ha ocurrido lo mismo en lo que se refiere a aquellos relativos a las medidas de protección comercial (Maruyama, 2021: 199). Con el bloqueo del OA se rompía el sistema de contrapesos establecido por el ESD, que forma parte del Acuerdo de Marrakech, como decimos, la actual rule of law del sistema comercial internacional.

En el trasfondo de la actuación estadounidense subyace una crítica hacia una $\mathrm{OMC}$ que considera ha dejado de estar conducida por los Gobiernos (power based system) para pasar a estarlo por un rígido sistema de solución de diferencias (rules-based system) dirigido por el OA. En su «Agenda de 2018 sobre Política Comercial», la Administración Trump llega a afirmar que la $\mathrm{OMC}$ «en vez de ser un foro de negociación donde los países pueden desarrollar nuevas, y mejores, normas, ha estado a veces dominada por un sistema de solución de diferencias donde "jueces" activistas han tratado de imponer sus propias preferencias políticas sobre los Estados miembros ${ }^{8}$. Desde el punto de vista estadounidense, el activismo judicial del OA conllevaría la falta de legitimidad de sus informes en la medida en que socava el principio básico de que son los Estados miembros de la OMC los que, a través de los órganos políticos de esta organización, tienen la competencia para legislar en materia de comercio internacional. Este tipo de críticas, de enorme calado, afectan a la misma esencia del mecanismo de solución de controversias de la OMC y a la dicotomía, no solo doctrinal, entre una power-oriented diplomacy vs. una rule-oriented diplomacy (Jackson et al., 1995).

\section{EL INFORME LIGHTHIZER}

En la evolución de acontecimientos que ha culminado con la crisis del SSD de la OMC es esencial hacer mención al informe presentado en febrero de 2020 por el representante de Comercio de los EE. UU., R. E. Lighthizer? Se trata del primer trabajo de esta naturaleza efectuado desde la Administración estadounidense para sintetizar las críticas al SSD de la OMC y, en particular, al OA.

8 Office of the United States Trade Representative, 2018 Trade Policy on the Trade Agreements Program, p. 2.

9 United States Trade Representative, Report on the Appellate Body of the World Trade Organization, 2020. 
Algunas de las críticas que recoge el informe Lighthizer ya habían sido formuladas en el OSD por EE. UU. con anterioridad a la llegada al poder de Donald Trump. Así, durante la presidencia de George W. Bush EE. UU. propuso conjuntamente con Chile la mejora del ESD con objeto de incrementar el control de los miembros de la OMC sobre el proceso de adopción de los informes (Hoekman y Mavroidis, 2019a: 113) ${ }^{10}$. Durante la de Barack Obama, como hemos señalado, se dio un paso más al impedir la renovación de los mandatos de determinados miembros del OA. Sin embargo, la novedad de la Administración Trump radicó en la adopción de una estrategia concreta consistente en el bloqueo del OA, al impedir la renovación de sus miembros, incumpliendo, de este modo, sus obligaciones derivadas del ESD (arts. 3, 17 y 23.1) y la rule of law del sistema económico internacional (Petersmann, 2018a: 112 y 121).

Es indudable que, al decidir asumir este papel, EE. UU. se sitúa en las antípodas de lo que ha sido su tradicional política impulsora de la gobernanza económica internacional, y que quedó reflejada, en particular, en su protagonismo durante la ronda de Uruguay del GATT que concluyó con la creación de la OMC y su complejo normativo, incluyendo el ESD (Hoekman y Mavroidis, 2019b; Sacerdoti, 2019: 37). La pregunta que debemos formularnos es por qué lo hizo. Y es aquí donde entran en juego el conjunto de críticas que el informe Lighthizer trata de sistematizar.

A modo de síntesis, las principales críticas de la Administración estadounidense respecto a la actuación del OA recogidas en este informe pueden ser clasificadas en tres categorías. En primer lugar, figuran las críticas de carácter procedimental. Hay una segunda categoría de acusaciones que tienen una mayor carga de profundidad, y que cabe calificar como críticas de carácter sistémico. Por último, el informe cuestiona la interpretación y consiguiente aplicación por parte del OA de determinadas disposiciones de los acuerdos de la OMC relativos a las medidas de protección comercial, concretamente las relativas al dumping, a las subvenciones y, por último, a las salvaguardias. A estas últimas las denominaremos "críticas de carácter sustantivo».

\section{CRÍTICAS DE CARÁCTER PROCEDIMENTAL}

Dentro de la categoría de críticas de carácter procedimental el informe Lighthizer destaca, en primer lugar, lo que la doctrina califica como la «cuestión del plazo de los noventa días». Concretamente, se cuestiona el incumplimiento por parte del OA de los plazos establecidos en el art. 17. 5

10 TN/DS/W/28, 23.12.2002. 
del ESD para la adopción de los informes. Este artículo establece que, «por regla general, la duración del procedimiento entre la fecha en que una parte en la diferencia notifique formalmente su decisión de apelar y la fecha en que el OA distribuya su informe no excederá de sesenta días», si bien contempla una posible ampliación de ese plazo hasta los noventa días «si el OA considera que no puede rendir su informe dentro de los sesenta días, debiendo comunicar al OSD por escrito los motivos del retraso, indicando el plazo en el que estima que podrá presentarlo». En ningún caso, añade, la duración del procedimiento excederá de noventa días. El informe Lighthizer considera que el límite del plazo de los noventa días es categórico y no admite excepción, lo que impediría que el OA pueda emitir informes más allá del término de los noventa días. Sin embargo, también recuerda que, desde 2011, el OA, de modo rutinario, ha ignorado este plazo, lo que mina la confianza en el sistema y promueve el que las prácticas comerciales desleales continúen desplegando sus efectos nocivos durante estos procedimientos así alargados (cabe recordar en este punto que en el SSD de la OMC no cabe la adopción de medidas cautelares). El informe también afirma que ello conduce al OA a abordar cuestiones innecesarias para resolver las diferencias, dando lugar a indeseables opiniones consultivas. De este modo, lo que inicialmente se presenta como un incumplimiento del ESD por parte del OA de carácter procedimental acabaría afectando a cuestiones de carácter sustantivo.

A continuación, el informe Lighthizer aborda la denominada «cuestión relativa a la Regla 15 de los Procedimientos de trabajo para el examen en apelación de la OMC». Es importante señalar que tales «Procedimientos de trabajo» fueron aprobados en 1995 por el OA en consulta con el director general de la OMC y el presidente del OSD ${ }^{11}$ (Pasoyova et al., 2018:3). Según la Regla 15: «Una persona que deje de ser miembro del OA podrá, con autorización del OA y previa notificación al OSD, terminar la sustanciación de cualquier apelación a la que hubiera sido asignada cuando era miembro y, a tal efecto únicamente, se considerará que sigue siendo miembro del $\mathrm{OA} »^{12}$. Pues bien, EE. UU. pone en duda la legalidad de la Regla 15 y considera que el OA no tiene autoridad para permitir que sus miembros puedan culminar el trabajo pendiente en apelación cuando su mandato ha expirado. Por consiguiente, cuestiona el que determinados miembros del OA —así Ramírez y Van den Bossche- hayan continuado trabajando en asuntos pendientes a pesar de la expiración de su mandato. Todo ello supondría para EE. UU. una vulneración del art. 17.2 del ESD, según el cual: «El OSD nombrará por un periodo de

$11 \mathrm{WT} / \mathrm{AB} / \mathrm{WP} / 6,16.8 .2010$.

12 Id. 
cuatro años a las personas que formarán parte del OA y podrá renovar una vez el mandato de cada una de ellas».

En tercer lugar, figura la llamada "cuestión del derecho interno". Concretamente, el informe considera que el OA se ha extralimitado al revisar cuestiones de hecho, función que, según los arts. 11 y 17. 6 del ESD, corresponde en exclusiva a los grupos especiales. Según el art. 11: "Cada grupo especial deberá hacer una evaluación objetiva del asunto que se le haya sometido, que incluya una evaluación objetiva de los hechos». En este mismo sentido, el art. 17. 6 del ESD señala que: «La apelación tendrá únicamente por objeto las cuestiones de derecho tratadas en el informe del grupo especial y las interpretaciones jurídicas formuladas por éste». En relación con esta cuestión, la doctrina ha señalado que, si bien los hechos no están sometidos a revisión por parte del OA en virtud del art. 17. 6 del ESD, este puede revisar la objetividad de la evaluación de los hechos efectuada por parte de un grupo especial a la luz del art. 11 del ESD, incluyendo su apreciación de las pruebas (Malacrida y Marceau, 2018: 39). El informe Lighthizer también cuestiona el que el $\mathrm{OA}$ se pronuncie en relación al derecho interno de los miembros de la OMC, lo cual conllevaría igualmente una violación del art. 17. 6 del ESD.

\section{CRÍTICAS DE CARÁCTER SISTÉMICO}

Las críticas de carácter sistémico tienen una mayor carga de profundidad en la medida en que ponen en tela de juicio el papel del OA en relación con la interpretación y aplicación de las normas de la OMC, incluido el ESD. Entre estas críticas encontramos, en primer lugar, la denominada «cuestión relativa al activismo judicial del OA». En particular, EE. UU. considera que el OA interpreta de modo extensivo las normas comerciales, lo que entraña un incremento de los derechos establecidos por los acuerdos abarcados (judicial over-reach), en violación del art. 3. 7 del ESD, que establece como objetivo del mecanismo de solución de diferencias el hallar una solución positiva a las diferencias que esté en conformidad con los acuerdos abarcados; y del IX. 2 del Acuerdo de la OMC, que otorga el poder de efectuar una interpretación auténtica de los acuerdos abarcados a los miembros de la OMC. En efecto, según este último artículo: «La Conferencia Ministerial y el Consejo General tendrán la facultad exclusiva de adoptar interpretaciones del presente Acuerdo y de los Acuerdos Comerciales Multilaterales». Asimismo, cuestiona el que determinados informes del OA aborden cuestiones innecesarias o superfluas (obiter dicta), esto es, no planteadas por las partes en la diferencia.

Dentro de esta segunda categoría de críticas se puede incluir también la denominada "cuestión de los precedentes». Concretamente, el informe se muestra en abierto desacuerdo con el carácter de precedente vinculante que 
el OA otorga a sus informes, lo que contribuye a crear una suerte de «jurisprudencia» comercial internacional (de facto stare decisis). Para EE. UU. ello contradice el texto de los acuerdos de la OMC así como la voluntad de las partes contratantes, para quienes cualquier cambio que afecte a sus derechos y obligaciones exige su previo consentimiento. Es preciso recordar que, en relación con esta cuestión, el OA ha considerado que, si bien la interpretación efectuada por los órganos de solución de diferencias se limita a las partes y a la materia objeto de la diferencia (art. 3.9 ESD), ello no impide, en aras de lograr una mayor coherencia, seguir el razonamiento contenido en previos informes. Pero la cuestión dista mucho de pacífica y ni siquiera es compartida en todos los casos por los grupos especiales. Así, por ejemplo, como decimos, mientras que el $\mathrm{OA}$ se ha encargado de afirmar que «seguir las conclusiones a que ha llegado el OA en diferencias anteriores no solo es apropiado, sino que es precisamente lo que se espera de los grupos especiales, sobre todo cuando las cuestiones son las mismas» ${ }^{13}$, el grupo especial que resolvió el asunto India-Protección mediante patente de los productos farmacéuticos señaló que: «Las decisiones anteriores de grupos especiales o del OA no vinculan a los grupos especiales, aunque esas decisiones se refieran a la misma cuestión» ${ }^{14}$. Las divergencias entre ambas instancias de solución de controversias han sido especialmente evidentes en relación con la aplicación del Acuerdo antidumping, de lo que es exponente el asunto Estados Unidos - Medidas antidumping que aplican el método de fijación de precios diferenciales a la madera blanda procedente del Canadá, en el que se debatía ante un grupo especial la técnica nacional de determinación del dumping conocida como «reducción a cero» (zeroing), que este se negó a considerar incompatible con el Acuerdo antidumping, pese a que así lo había prescrito previamente el $\mathrm{OA}^{15}$. De modo que el informe Lighthizer, en este punto, se inclinaría hacia la posición que defiende el panel. El informe llega a acusar a determinados miembros de la $\mathrm{OMC}$ de intentar lograr en el OA lo que no pueden alcanzar en una mesa de negociación («Some Members seek to obtain throuh a "binding” Appellate Body interpretation what they could not achieve through negotiation»).

13 OMC, Estados Unidos-Examen por extinción de las medidas antidumping impuestas a los artículos tubulares para campos petroliferos procedentes de Argentina, WT/DS268/ $\mathrm{AB} / \mathrm{R}$, de 17.12.2004, nota 24, párr. 188.

14 OMC, India-Protección mediante patente de los productos farmacéuticos y los productos quimicos para la agricultura, WT/DS79/R, de 24.8.1998, párr. 7.30.

15 OMC, Estados Unidos - Método de fijación de precios diferenciales a la madera blanda, WT/DS534/R, de 9.4.2019, especialmente párrs. 7-107. 
En tercer lugar, el informe Lighthizer critica que el OA se haya arrogado poder discrecional para emitir (o no) sus recomendaciones, en violación del art. 19. 1 del ESD, que le obliga en todo caso a emitir un informe que incluya tales recomendaciones. También entiende que el OA se ha extralimitado al opinar sobre cuestiones que son competencia de otros órganos de la OMC, como lo son la Conferencia Ministerial, el Consejo General o el OSD.

\section{CRÍTICAS DE TIPO SUSTANTIVO}

La última categoría de críticas del informe Lighthizer, las de carácter sustantivo, van dirigidas al modo en el que el OA ha interpretado, y consiguientemente aplicado, determinadas disposiciones de los acuerdos de la OMC relativos a las medidas de protección comercial, esto es, dumping, subvenciones y salvaguardias. Así, su interpretación restrictiva del concepto «organismo público» del art. 1.1 del Acuerdo sobre subvenciones y medidas compensatorias; la interpretación errónea del art. 17. 6 del Acuerdo antidumping, una disposición que, según los EE. UU., salvaguarda el poder de apreciación del que disfrutan las autoridades administrativas internas en la interpretación de las disposiciones del mencionado acuerdo, y que no habría sido tenida en cuenta por el OA al impedir que sean tales autoridades quienes determinen los márgenes de dumping según la técnica denominada zeroing (que permite el incremento de tales márgenes al no tener en cuenta los márgenes individuales de dumping negativos) dando lugar a las divergencias interpretativas anteriormente señaladas; o la interpretación libre dada por el OA al art. XIX del Acuerdo sobre salvaguardias, al establecer requisitos adicionales para la imposición de las medidas a los establecidos por el mencionado artículo.

Aunque figuren en último lugar en el informe estadounidense, las críticas de carácter sustantivo explicarían la naturaleza de la amenaza que para EE. UU. simboliza el OA como un órgano que ha asumido de facto un papel que va más allá de lo que le asigna el ESD. En este sentido, Hoekman considera que habría sido mejor que los EE. UU. hubiesen puesto el acento en la necesidad de abordar este tipo de preocupaciones de carácter sustantivo en lugar de optar por la acción drástica de dinamitar el funcionamiento del OA (Hoekman, 2016). Sin embargo, es necesario también recordar la dificultad inherente al proceso de reforma del derecho de la OMC, debido a la regla del consenso positivo, lo que posiblemente influyó en que la administración estadounidense decidiera echar el freno de mano que representa el bloqueo del OA, impidiendo el nombramiento de nuevos miembros. 


\section{PROPUESTAS DE DESBLOQUEO DEL SISTEMA DE SOLUCIÓN DE DIFERENCIAS DE LA OMC}

\section{LA INICIATIVA DE LA UE PARA DESBLOQUEAR LA PARÁLISIS DEL SSD}

Un año antes de que se produjera el bloqueo del OA, el Consejo Europeo de 28-29 de junio de 2018 pidió a la Comisión de la UE que propusiera un planteamiento de conjunto para lograr, en colaboración con otros socios de la $\mathrm{OMC}$, «una solución de diferencias más efectiva y transparente». En cumplimiento de este mandato, el 18 de septiembre de ese año la Comisión adoptó un informe en el que formuló propuestas concretas con objeto de llegar a un acuerdo para resolver la situación existente ${ }^{16}$.

En este documento (Concept Paper) la Comisión describe a la UE como una firme defensora del sistema comercial multilateral, y afirma que la OMC es indispensable para asegurar un comercio libre y justo, si bien considera que no ha sido capaz de adaptarse a un mundo que ha cambiado («in essence, since 1995 the world has changed; the WTO has not»). Sus propuestas respecto a la solución de diferencias parten de un análisis global de los factores que han propiciado el actual bloqueo del OA, con especial atención a las críticas estadounidenses recogidas en la Trade Policy Agenda de los EE. UU. de 2018. En particular, se mencionan las relativas a la Regla 15, la cuestión de los noventa días, la cuestión de las opiniones consultivas innecesarias para resolver la diferencia, la cuestión del derecho interno, la cuestión de los precedentes, e incluso las críticas de carácter sustantivo que luego serían recogidas en el informe Lighthizer, a las que hemos hecho referencia anteriormente. Ello demuestra, desde mi punto de vista, que las preocupaciones de EE. UU. respecto al funcionamiento del OA son en gran medida compartidas por la UE, y que lo que diferencia a la UE respecto a EE. UU. es el papel de crítica constructiva al sistema que esta asume, así como su denodado esfuerzo por liderar un proceso capaz de restablecer la rule of law.

La UE fija su posición estableciendo como primer paso la necesidad de acometer una modificación de las disposiciones del ESD relativas al funcionamiento del OA. Ahora bien, dado que las cuestiones abordadas en este documento exigen la formulación de enmiendas al art. 17 del ESD, relativo al examen en apelación, la UE propone que sea la Conferencia Ministerial quien las adopte por consenso de conformidad con el art. X: 8 del Acuerdo de la OMC, si bien afirma que también podría hacerlo el Consejo General

16 Comisión Europea, Comunicado de prensa de 26 de noviembre de 2018. El Concept Paper figura como documento anexo a dicho comunicado. 
de la OMC puesto que tiene capacidad de decisión en los intervalos entre reuniones de la Conferencia Ministerial.

En noviembre de 2018 la UE llegó a presentar una propuesta, con el copatrocinio de otros miembros de la OMC, ante el Consejo General de la OMC. Esta propuesta aborda las preocupaciones estadounidenses en relación con el funcionamiento del OA de la OMC tanto de carácter procedimental como sistémico, pero omite las de carácter sustantivo, una cuestión que se deja para una etapa posterior a la del restablecimiento del funcionamiento del OA. Concretamente, las propuestas formuladas por la UE y el resto de países patrocinadores tienen como objetivo: a) garantizar que los procedimientos de apelación respeten el plazo de los noventa días fijado en el ESD, a menos que las partes en la diferencia acuerden lo contrario («cuestión del plazo de noventa días»); b) establecer nuevas normas que aclaren en qué casos los miembros del OA cuyo mandato ha expirado pueden permanecer conociendo de un asunto con el fin de completar el procedimiento en curso («cuestión relativa a la Regla 15»); c) indicar que el OA debería tratar únicamente aquellas cuestiones que sean necesarias para resolver la diferencia ("cuestión del activismo del $\mathrm{OA}$ ») d) aclarar que, entre los asuntos jurídicos de los que conoce el OA, no se incluye el sentido de la legislación nacional (conocido como «la cuestión del derecho interno»); y e) organizar reuniones anuales (en el marco del OSD) entre los miembros de la OMC y el OA con el objeto de debatir tendencias y cuestiones sistémicas, tales como la preocupación expresada por EE. UU. respecto al "enfoque» del OA de considerar sus informes como precedentes que deben seguir los grupos especiales a menos que existan «razones imperativas» («la cuestión de los precedentes») ${ }^{17}$. Sin embargo, no se logró el consenso necesario para llevarlas a efecto por falta de voluntad política por parte de los EE. UU. ${ }^{18}$.

17 Id.

18 Comunicación ante el Consejo General de la OMC por parte de la Unión Europea, Australia, China, Canadá, la República de Corea, India, Islandia, Méjico, Nueva Zelanda y Singapur (WT/GC/W/752, de 26.11.2018). A esta comunicación le sucedieron otras que incorporaron a nuevos firmantes (Costa Rica, Montenegro, Noruega y Singapur, WT/GC/W752/Rev.2, de 11.12.2018), y modificaciones adicionales [Unión Europea, China, India y Montenegro, (WT/GC/753/Rev.1, 11.12.2018)], así como otras comunicaciones por parte de países o grupos de países: a) Australia, Singapur, Costa Rica, Canadá y Suiza (WT/GC/754/Rev.2, 11.12.2018); b) Honduras (WT/GC/W/760, 21.1.2010); c) Brasil, Paraguay y Uruguay (WT/GC/767/ Rev.1, 25.1.2019); Japón, Australia y Chile (WT/GC/W/768/Rev.1, 26.4.2019); y Tailandia (WT/GC/W/769, 26.4.2019). 


\section{EL PROCESO INFORMAL SOBRE CUESTIONES RELACIONADAS CON EL FUNCIONAMIENTO DEL ÓRGANO DE APELACIÓN}

Paralelamente, en el Consejo General de la OMC del 12 de diciembre de 2018, los miembros de la OMC aceptaron la propuesta de su presidente, Junichi Ihara, de iniciar un proceso informal para superar el estancamiento en la selección de los miembros del OA. A tal fin fue designado el embajador de Nueva Zelanda ante la OMC, David Walker, como facilitador del denominado "Proceso informal sobre cuestiones relacionadas con el funcionamiento del OA». Walker se afanó en propiciar un "debate centrado en las soluciones» en el que se pudieran manifestar las posiciones de los miembros de la OMC a este respecto. El proceso incluyó diversas reuniones que adoptaron diferentes formatos. Así: a) reuniones abiertas a todos los miembros de la OMC; b) reuniones en un grupo reducido de delegaciones que habían: i) presentado propuestas por escrito; ii) planteado preocupaciones por el debido funcionamiento del OA y su respeto de las normas de la OMC; iii) formulado propuestas alternativas; $y / o$ iv) planteado preguntas complementarias o inquietudes en relación con las propuestas o cuestiones de que se trata; y por último, c) reuniones informales. El Consejo General de la OMC fue informado regularmente de los avances alcanzados en este proceso informal ${ }^{19}$.

Walker acabaría presentando al Consejo General de la OMC de 15 de octubre de 2019 un informe que recogía los "puntos de convergencia» en relación con el funcionamiento de la $\mathrm{OMC}^{20}$. Las propuestas del embajador Walker concuerdan en gran medida con las formuladas ante la OMC por la UE y otros países, anteriormente señaladas, especialmente en lo que concierne a "la cuestión del plazo de noventa días», "la cuestión del derecho interno», la «cuestión del activismo del OA» o «la cuestión de los precedentes» ${ }^{21}$.

$19 \mathrm{JOB} / \mathrm{GC} / 215,1.3 .2019, \mathrm{JOB} / \mathrm{GC} / 217$, 8.5.2019, JOB/GC/220, 25.7.2019.

$20 \mathrm{JOB} / \mathrm{GC} / 222,15.10 .2019$.

21 Concretamente son las siguientes: a) la obligación del OA de emitir sus informes a más tardar noventa días después de la fecha en que una parte en la diferencia notifique su intención de apelar a menos que, en relación con asuntos de inusual complejidad, las partes acuerden con el $\mathrm{OA}$ ampliar el plazo de emisión del informe; b) entre los asuntos jurídicos de los que conoce el $\mathrm{OA}$ no se incluye el sentido de la legislación nacional; el ESD no autoriza al OA a llevar a cabo un examen «ex novo» ni a "completar el análisis» de los hechos de una diferencia; c) los miembros que participan en procedimientos de apelación deben abstenerse de presentar argumentos extensos e innecesarios con la intención de revocar las constataciones fácticas en apelación; d) el OA no puede resolver o decidir sobre cuestiones que ninguna de las partes ha planteado; e) no se crean precedentes vinculantes a través del procedimiento de solución 
Posteriormente estas propuestas fueron recogidas en un proyecto de decisión titulado «Funcionamiento del Órgano de Apelación», que fue presentado ante el Consejo General de la $\mathrm{OMC}^{22}$ pero que no fue adoptado en dicho órgano debido a la falta de consenso ${ }^{23}$. La doctrina ha señalado como posible causa de este fracaso el hecho de que el informe Walker no recogiese las críticas estadounidenses de carácter sustantivo, esto es, las relativas a la interpretación por parte del OA de los acuerdos de la OMC relativos a las medidas de protección comercial (Maruyama, 2021: 197).

\section{EL ACUERDO MULTILATERAL DE ARBITRAJE DE APELACIÓN}

Una vez que se produjo el bloqueo del OA, y ante la imposibilidad de alcanzar un acuerdo con EE. UU. en torno a las propuestas anteriormente indicadas, la UE auspició (De Andrade, 2019: 121) la celebración del Acuerdo Multilateral de Arbitraje de Apelación (Multi-Party Interim Appeal Arbitration Arrangement pursuant Article 25 of the DSU, MPIA, en adelante). Este acuerdo contempla un sistema alternativo de solución de diferencias comerciales basado en el arbitraje previsto en el art. 25. 1 del ESD. Cabe recordar que, según dicho art., cuya inclusión en el ESD fue casualmente una propuesta estadounidense (Andersen et al., 2017: 2; Bacchus, 2018: 26): «Un procedimiento rápido de arbitraje en la $\mathrm{OMC}$ como medio alternativo de solución de diferencias puede facilitar la resolución de algunos litigios que tengan por objeto cuestiones claramente definidas por ambas partes ${ }^{24}$.

La sumisión al arbitraje, de este modo, se basa en el MPIA, que fue notificado a la $\mathrm{OMC}^{25}$ y está en vigor desde el 30 de abril de 2020. Hasta la fecha veinticuatro delegaciones han manifestado su voluntad de ser partes

de diferencias de la OMC; f) las constataciones y recomendaciones efectuadas en el marco del SSD de la OMC no pueden entrañar el aumento o la reducción de los derechos y obligaciones establecidos en los acuerdos abarcados; y g) la creación de un mecanismo de diálogo periódico entre los miembros de la OMC y el OA (doc. JOB/ $\mathrm{GC} / 222,15.10 .2019)$.

22 WT/GC/W/791, 28.11.2019.

23 OMC, Informe Anual, 2020, p. 61.

$24 \mathrm{El}$ arbitraje del art. 25 del ESD ha sido escasamente utilizado en el pasado. En este sentido, únicamente se puede mencionar el laudo arbitral dictado en el asunto Estados Unidos-artículo 110 (5) de la Ley de derecho de autor de los Estados Unidos (WT/ DS160/ARB25/1, de 9.11.2001).

25 Declaración sobre un mecanismo para elaborar, documentar y comunicar prácticas y procedimientos en la sustanciación de diferencias en la OMC (JOB/DSB/1/Add.12). 
en el MPIA ${ }^{26}$. En el anexo al MPIA figura el procedimiento de acuerdo de arbitraje a seguir para la apelación de conformidad con las disposiciones contenidas en un Anexo a la Declaración remitida a la OMC. Su anexo II regula la composición de la lista de árbitros ${ }^{27}$. La doctrina especializada ha querido ver en esta posibilidad de que las partes en una diferencia puedan optar por el arbitraje del art. 25 de ESD un reflejo de las normas del derecho internacional relativas a la libertad de elección de los medios de solución de diferencias y, en particular, con el art. 33 de la Carta de las Naciones Unidas (Carreau y Juillard, 2017: 131).

El MPIA está concebido con carácter provisional («hasta que el mecanismo de apelación de la OMC vuelva a funcionar») y abierto a la participación de terceros («cualquier Miembro de la OMC puede adherirse al mecanismo en cualquier momento»). Lo esencial de este acuerdo ha sido contemplar, prospectivamente, la posibilidad de que los países que participan en él (sus «miembros participantes») puedan, una vez obtenido el informe del grupo especial, solicitar un arbitraje ante el denominado Órgano de Apelación Provisional para la solución de diferencias (OAP), en lugar de acudir al OA de la OMC. Además, los miembros participantes en el acuerdo se comprometen a no interponer recursos de apelación ante el OA de la OMC. Como decimos, los diez miembros que componen el OAP ya han sido designados por los miembros del MPIA, estando previsto que, en cada caso que se les someta, actúen tres de ellos. Su designación para resolver una diferencia particular se basará en el principio de rotación. La implicación de los órganos de la OMC se hace patente en el hecho de que sea el director general de la OMC quien notifique a las partes y los terceros los resultados de la selección. Por otra parte, el acuerdo prevé que los denominados «árbitros de apelación» cuenten con asistencia administrativa y jurídica, "totalmente separada del personal de la Secretaría de la OMC», si bien «los miembros participantes solicitan al director general de la OMC que se asegure de la disponibilidad de una estructura de apoyo».

El arbitraje previsto en el MPIA se basa en el derecho de la OMC, con algunas especificidades. El propio acuerdo de arbitraje señala que el arbitraje

26 Son: la Unión Europea y los siguientes Estados: Australia, Benín, Brasil, Canadá, China, Chile, Colombia, Costa Rica, Ecuador, Guatemala, Hong Kong China, Islandia, Méjico, Montenegro, Nicaragua, Noruega, Nueva Zelanda, Paquistán, Singapur, Suiza, Ucrania y Uruguay.

27 Son los siguientes: Mateo Diego-Fernández Andrade, Thomas Cottier, Locknie Hsu, Valerie Hughes, Alejandro Jara, José Alfredo Graça Lima, Claudia Orozco, Joost Pauwelyn, Penélope Ridings, Guohua Yang (JOB/DSB/1/Add.12/Suppl.5). 
se regirá por las disposiciones del ESD y demás normas y procedimientos aplicables al examen en apelación (apdo. 11 del procedimiento arbitral en apelación). Por consiguiente, se adapta a lo establecido en el art. 3. 5 del ESD, que establece que: "Todas las soluciones de los asuntos planteados formalmente con arreglo a las disposiciones en materia de consultas y solución de diferencias de los acuerdos abarcados, incluidos los laudos arbitrales, habrán de ser compatibles con dichos acuerdos y no deberán anular ni menoscabar las ventajas resultantes de los mismos para ninguno de sus Miembros, ni deberán poner obstáculos a la consecución de ninguno de los objetivos de dichos acuerdos».

A diferencia de los informes emitidos por los grupos especiales y del OA, que necesitan ser adoptados por el OSD para devenir obligatorios según lo establecido en el art. 25. 3 del ESD, los laudos arbitrales son obligatorios para las partes y deben ser notificados al OSD y al consejo o comité de los acuerdos pertinentes, en los que cualquier miembro podrá plantear cualquier cuestión con ellos relacionada. Dicho artículo también establece, en su apdo. 4, la posible aplicación a este procedimiento de arbitraje de los arts. 21 y 22 del ESD, sobre vigilancia y compensación por parte del OSD en caso de incumplimiento de los laudos arbitrales. El acuerdo de arbitraje en apelación puntualiza en esta línea que el laudo arbitral es definitivo, debiendo las partes acatarlo. De conformidad con el párr. 3 del art. 25 del ESD, «el laudo será notificado a, pero no adoptado por, el OSD, y al Consejo o Comité de acuerdos pertinentes» (apdo. 15).

En el anexo al MPIA figura un modelo de «Procedimiento convenido para el arbitraje previsto en el artículo 25 del ESD en la diferencia DS X», que ya se ha utilizado en los arbitrajes solicitados al amparo del MPIA ${ }^{28}$. Se prevé que, después del traslado del informe del grupo especial a las partes, pero a más tardar diez días antes de la fecha prevista de distribución del informe definitivo del grupo especial al resto de los miembros, cualquiera de las partes pueda solicitar que el grupo especial suspenda el procedimiento, entendiendo, además, que la petición formulada en este sentido por cualquiera de las partes que participan en el MPIA constituye una solicitud conjunta. El procedimiento

28 OMC, Canadá-Medidas relativas al Comercio de aeronaves comerciales, Comunicación de 20.5.2020 (WT/DS522/20). El arbitraje se ha suspendido tras la decisión de Brasil de retirar su reclamación (WT/DS522/23); OMC, Canadá-Medidas que rigen la venta de vino, Comunicación de 3.6.2020 (WT/DS537/15). Este caso ha finalizado mediante solución mutuamente convenida (WT/DS537/18); OMC, Colombia-Derechos antidumping sobre las patatas (papas) fritas congeladas procedentes de Bélgica, Alemania y los Países Bajos, Comunicación de 22.4.2021 (WT/DS591/3/Rev.1). 
de arbitraje en apelación se inicia con el anuncio de apelación a la Secretaría de la OMC, a más tardar veinte días después de que sea efectiva la suspensión del procedimiento del grupo especial. Cabe desistimiento en cualquier momento del arbitraje mediante notificación a los árbitros.

La voluntad de los miembros que participan en el arbitraje de apelación de tener en cuenta las críticas estadounidenses respecto al funcionamiento del OA se hace patente en algunos apartados del procedimiento regulado en el anexo al MPIA. Así, el hecho de que: a) la apelación solo tenga por objeto las cuestiones de derecho tratadas en el informe del grupo especial y las interpretaciones jurídicas formuladas por este (apdo. 9 del procedimiento); b) los árbitros solo examinen las cuestiones que sean necesarias para la solución de la diferencia y aquellas cuestiones que hayan sido planteadas por las partes, «sin perjuicio de su obligación de pronunciarse sobre cuestiones jurisdiccionales» (apdo. 10 del procedimiento); c) los árbitros dicten el laudo dentro de los noventa días siguientes a la presentación del anuncio de apelación (apdo. 12), si bien se permite que, a propuesta de los árbitros, las partes puedan convenir en ampliar el plazo de noventa días para la emisión del laudo; y d) en caso necesario para dictar el laudo en el plazo de los noventa días, los árbitros podrán proponer a las partes medidas sustantivas, como la exclusión de las alegaciones basadas en una supuesta falta de evaluación objetiva de los hechos de conformidad con el art. 11 del ESD (apdo. 13). También el MPIA es claro cuando señala que: «Los laudos arbitrales no podrán entrañar el aumento o la reducción de los derechos y obligaciones establecidos en los acuerdos abarcados» (párr. sexto del preámbulo).

Sin embargo, el MPIA no aborda la cuestión de la aplicación de la Regla 15, referida en este caso a los árbitros, lo que deja en el aire si estos pueden continuar trabajando en un asunto pendiente después de la expiración de su mandato. Y es ambiguo en lo que concierne a la "cuestión de los precedentes», cuestión esta que es abordada solo de forma indirecta (De Andrade, 2019: 140). Así, cuando en el MPIA se afirma que «los Miembros atribuyen un gran valor a la coherencia y previsibilidad en la interpretación de los derechos y obligaciones en el marco de los acuerdos abarcados» (párr. sexto del preámbulo del MPIA). En esta misma línea podría interpretarse el que los árbitros se rijan por el principio de colegialidad, pudiendo debatir sus decisiones relativas a la apelación con todos los demás miembros de la lista de árbitros, sin perjuicio de su exclusiva responsabilidad y libertad respecto a esas decisiones, lo que implica el que todos los miembros de la lista de árbitros reciban todos los documentos relativos a la apelación (apdo. 8 del procedimiento). A pesar de ello, se puede afirmar que en el MPIA no queda del todo claro el peso que los árbitros en apelación deben dar a los informes pasados de los grupos especiales o del OA, o incluso del propio OAP. 


\section{LA APLICACIÓN DE CONTRAMEDIDAS UNILATERALES POR PARTE DE LA UE EN RELACIÓN CON PAÍSES NO PARTICIPANTES EN EL MPIA}

El deseo de la UE por cumplir el objetivo del multilateralismo y la búsqueda de soluciones globales no ha impedido el que esta se arrogue capacidad para adoptar contramedidas unilaterales en relación con los países que han decidido no participar en el MPIA. En este sentido, la reciente reforma del art. 5 del Reglamento (UE) 2021/167 del Parlamento Europeo y del Consejo permite que la UE adopte contramedidas en relación con las diferencias comerciales entre la UE y miembros de la OMC no participantes en el MPIA en las que un grupo especial haya adoptado un informe favorable a las reclamaciones presentadas por la UE, pero el proceso de solución de diferencias de la OMC se encuentre bloqueado debido a la presentación por la otra parte en la controversia de un recurso de nulo de pleno derecho ante el OA, y no se llegue a un acuerdo con ella sobre la posibilidad de recurrir al arbitraje provisional en apelación previsto en el art. 25 del ESD ${ }^{29}$.

Estas contramedidas pueden consistir en derechos de aduana, restricciones cuantitativas sobre las importaciones o exportaciones de mercancías y medidas en el ámbito de la contratación pública. La suspensión de obligaciones para con la otra parte que implican las contramedidas abarca no solo al comercio de mercancías sino también al comercio de servicios y a los aspectos de los derechos de propiedad intelectual relacionados con el comercio. El reglamento también posibilita la adopción de contramedidas cuando un socio en un acuerdo comercial bilateral o regional no tome las medidas necesarias para que funcione el mecanismo de solución de diferencias previsto en dichos acuerdos, o cuando dilate de forma indebida el procedimiento.

Es evidente que la adopción de estas contramedidas unilaterales por parte de la UE vulnera el art. 23 del ESD, que identifica al OSD de la OMC como único órgano capacitado para autorizar la adopción de las contramedidas. Sin embargo, considero que la ilicitud de tales contramedidas quedaría excluida, a la luz del derecho internacional, según lo dispuesto en el art. 22 (titulado "Contramedidas en razón de un hecho internacionalmente ilícito») del Proyecto de artículos de la Comisión de Derecho Internacional (CDI, en adelante) sobre responsabilidad del Estado por hechos internacionalmente ilícitos, considerado expresión del derecho consuetudinario. En efecto, cabe interpretar que la obligación, contenida en el art. 52. 3 b) del Proyecto de

29 Art. 5 del Reglamento (UE) 2021/167 del Parlamento Europeo y del Consejo, sobre el ejercicio de los derechos de la Unión para aplicar y hacer cumplir las normas comerciales internacionales, DO L 49 de 12.2.2021, p. 1. 
artículos de la CDI, de no aplicar contramedidas cuando una controversia esté sometida a una corte o a un tribunal con facultades para dictar soluciones vinculantes para las partes no es de aplicación, de conformidad con el art. 52. 4 de dicho proyecto de artículos, "si el Estado responsable no aplica de buena fe los procedimientos de solución de controversias». De modo que, extrapolando dichas normas al SSD de la OMC, se podría llegar a calificar el comportamiento de la parte que decide recurrir el informe del grupo especial ante el OA planteando un recurso nulo de pleno derecho como un comportamiento de mala fe, lo que justificaría la aplicación de las contramedidas unilaterales por parte de la UE (Wei, 2020: 8). Ello supondría que el régimen especial de responsabilidad internacional establecido por el ESD cedería el paso a la aplicación de las normas internacional generales en materia de responsabilidad internacional, incluyendo las normas internacionales generales en materia de contramedidas, cuando un miembro de la $\mathrm{OMC}$ actúe de mala fe al bloquear indebidamente el SSD de la OMC, o plantee un recurso nulo de pleno derecho ante el OA, a sabiendas de que este órgano no puede operar por falta de miembros.

\section{EL SSD A LA LUZ DE LA REFORMA DE LA POLÍTICA COMERCIAL DE LA UE}

Los desarrollos posteriores en el seno de la UE demuestran que la solución que ofrece el MPIA, incluyendo también el reforzamiento de las medidas de reacción que constituyen las contramedidas unilaterales mencionadas, no menoscaba el deseo de la UE de liderar un proceso que conduzca al pleno restablecimiento del SSD de la OMC, como decimos, la rule of law del sistema comercial internacional. En este sentido, en la reciente comunicación de la Comisión Europea sobre la reforma de la política comercial de la UE, titulada «Revisión de la política comercial: una política comercial abierta, sostenible y asertiva ${ }^{30}$, la UE explicita su voluntad de liderar el proceso de reforma de la OMC con el objeto de «restablecer un sistema de solución de diferencias plenamente operativo en la OMC con un Órgano de Apelación reformado"; una reforma que «debe conservar la norma del consenso negativo, la independencia del OA y el papel fundamental de la solución de diferencias para proporcionar seguridad y previsibilidad al sistema multilateral de comercio». Ello pone de manifiesto implícitamente el carácter provisional con que la UE concibe el MPIA y su deseo de restablecer la operatividad del OA.

30 Comunicación de la Comisión de la UE «Revisión de la política comercial: una política comercial abierta, sostenible y asertiva» (COM (2021) 66 final, de 18.2.2021, p. 7. 
En el anexo a esta comunicación, la Comisión señala que «el sistema de solución de diferencias ha retrocedido de facto a los tiempos del GATT en que era posible bloquear los informes de los grupos especiales». Es por ello que la UE, al tiempo que expresa su preocupación al respecto, identifica algunas cuestiones en las que coincide con la posición estadounidense, lo que podría servir como base de negociación de cara a la reunión de la Conferencia Ministerial que tendrá lugar a finales de 2021. Las cuestiones mencionadas son las siguientes: a) los órganos jurisdiccionales deben ejercer la economía procesal y no están limitados por los «precedentes», si bien deben tener en cuenta fallos previos en la medida en que los consideren pertinentes a efectos de la diferencia que les ocupe; b) los grupos especiales son quienes juzgan los hechos y el papel del OA debe limitarse estrictamente a examinar las cuestiones jurídicas planteadas en apelación; y c) los plazos deben respetarse escrupulosamente debiendo, no obstante, adoptarse medidas apropiadas para que esto sea posible. Ello demuestra que la UE, aunque sigue sin abordar el conjunto de críticas formuladas por los EE. UU. en el informe Lighthizer, especialmente las de carácter sustantivo, persiste en la defensa de la restauración del OA en el marco de un reformado SSD de la OMC.

\section{REFLEXIONES FINALES}

Es demasiado pronto para afirmar que el SSD de la OMC ha muerto. En primer lugar, porque se han producido cambios políticos significativos tanto en la OMC — con la designación de una nueva directora general (Ngozi Okonjo) - como en la Presidencia de los EE. UU. — con la designación de Joe Biden como nuevo presidente del país, que cuenta con una nueva representante de comercio (Katherine Thai) en sustitución de Lighthizer- que podrían influir tanto en el proceso de reforma de la OMC como de su sistema de solución de diferencias.

Además, pese al bloqueo del OA, el sistema de los grupos especiales sigue funcionando, si bien, como decimos, aquellos informes que son recurridos ante un inexistente OA (como ha ocurrido en 2020 en cinco casos; OMC Informe anual, 2021: 142 y 143) no pueden ser resueltos ni adoptados por el OSD, quedando en un vacío legal, lo que justifica tanto las propuestas alternativas del tipo del MPIA basadas en un sistema de doble arbitraje como la posible aplicación de contramedidas unilaterales por parte de la UE sobre la base del recientemente reformado art. 5 del Reglamento (UE) 2021/167 del Parlamento Europeo y del Consejo.

La evolución de acontecimientos que hemos descrito revela que muchas de las críticas estadounidenses al OA son compartidas por la UE pero que 
EE. UU. ya no se conforma con una solución de tipo provisional como poner en marcha el funcionamiento del OA (o participar en el MPIA) si no hay un acuerdo global en relación con cuestiones de carácter sustantivo del derecho de la OMC. Y, en este punto, la necesidad de consenso, en este caso positivo, en el seno de la OMC para reformar su derecho impide resolver a corto plazo la situación. Se trata de conocer el alcance exacto que quieren dar, no ya el $\mathrm{OA}$, sino los propios miembros de la OMC a los acuerdos de la OMC sobre medidas de protección comercial, por no mencionar la necesidad de actualizar el derecho de la OMC en relación con el modo de adopción de decisiones, o la regulación en materias como medio ambiente o condicionalidad social. En definitiva, es necesario saber qué derecho comercial quieren los grandes actores del comercio internacional para poder aplicar a este nuevo derecho las exigencias derivadas de la rule of law. En palabras de Creamer, es necesario lograr un nuevo equilibrio político que permita reconstruir y dar legitimidad al sistema (Creamer 2019: 54).

En este proceso la UE está llamada a desarrollar un importante papel. De hecho, su función como garante del Estado de Derecho en el proceso de crisis que está sufriendo el SSD de la OMC, concretamente en relación con la función del OA, ya ha tenido dos manifestaciones principales. En primer lugar, mediante la presentación de propuestas ante los órganos de la OMC. En este apartado incluimos la propuesta presentada ante el Consejo General de la OMC, que contó con el copatrocinio de otros miembros de la OMC, de noviembre de 2018, y su apoyo al proceso informal sobre cuestiones relacionadas con el funcionamiento del OA dirigido por el embajador Walker en el seno del OSD de la OMC. En segundo lugar, mediante su impulso a la celebración del Acuerdo Multilateral de Arbitraje de Apelación.

Aunque la solución transitoria que ofrece el MPIA se adapta, en principio, al ESD y, por lo tanto, no exige que se lleven a cabo enmiendas a su texto, sin embargo plantea algunos interrogantes. Así: a) el hecho de que queden fuera de este mecanismo muchas diferencias y, en particular, las que atañen a los EE. UU. (Creamer, 2019: 55; Koekman y Mavroidis, 2019: 114); o b) el que los laudos arbitrales, cuyo valor es obligatorio, no necesiten ser adoptados formalmente por el OSD, lo que los sitúa en gran medida al margen del SSD de la OMC (Hillman, 2020: 9). Tampoco queda claro cómo se resolverá en el seno del OAP la importante cuestión de los precedentes, por no mencionar aquellos casos en que el OAP se enfrente a la interpretación de disposiciones que no han sido previamente interpretadas en el marco del actual SSD de la OMC. En cualquier caso, es evidente que la creación de esta doble instancia de arbitraje del MPIA dista mucho del mecanismo basado en los grupos especiales y el OA del SSD de la OMC, dado el carácter permanente que este último órgano tiene. Y tampoco resuelve el problema de fondo que es la falta 
de actualización del derecho comercial internacional, incluido el ESD, algo que solo pueden acometer los miembros de la OMC en su conjunto (Hauser, 2003: 224).

Más problemática es la posible adopción de contramedidas unilaterales por parte la UE a los terceros Estados no participantes en el MPIA, en aplicación del art. 5 del Reglamento (UE) 2021/167 del Parlamento Europeo y del Consejo, ya que ello contraviene abiertamente el art. 23 del ESD de la $\mathrm{OMC}$ - que no solo establece la obligatoriedad del mecanismo de solución de diferencias de la OMC sino que además identifica al OSD de la OMC como único órgano capacitado en dicha organización internacional para autorizar la adopción de las contramedidas-, si bien podrían considerarse contramedidas admisibles a la luz del derecho internacional consuetudinario contenido en el Proyecto de artículos de la Comisión de Derecho Internacional sobre responsabilidad internacional del Estado por hechos internacionalmente ilícitos.

En cualquier caso, la comunicación de la Comisión sobre la «Revisión de la política comercial: una política comercial abierta, sostenible y asertiva» deja clara la voluntad de la UE de liderar el proceso de reforma de la OMC con el objeto de «restablecer un sistema de solución de diferencias plenamente operativo en la OMC con un Órgano de Apelación reformado». Es de desear que así sea y que los cambios a nivel político a los que antes hemos aludido promuevan un clima de mayor confianza que posibilite la reforma del derecho de la OMC, incluido su sistema de solución de diferencias.

\section{Bibliografía}

Andersen, S., Friedbacher, T., Lau, C., Lockhart, N., Remy, J. Y. y Sandford, I. (2017). Using arbitration under article 25 of the DSU to ensure the availability of appeals. Ginebra: Graduate Institute Geneva. Disponible en: https://bit.ly/3oD1D9M. Bacchus, J. (2018). Might Unmakes Right. The American Assault on the Rule of Law in World Trade. Waterloo: Centre for International Governance Innovation Papers.

Bhala, R., Gantz, D. A., Keating, S. B., Witmer, E. y Wood, C. N. (2020). WTO Case Review 2018. Arizona Journal of International Trade \& Comparative Law, 37 (1), 49-136.

Carreau, D. y Juillard, P. (2017). Droit International Économique. Paris: Dalloz.

Creamer, C. D. (2019). Can International Law Recover? From the WTO's Crown Jewel to Its Crown of Thorns. American Journal of International Law, 113, 51-55. Disponible en: https://doi.org/10.1017/aju.2019.1.

De Andrade, M. (2019). Procedural innovations in the MPIA: A way to strengthen the WTO dispute settlement mechanism? Questions of International Law, Zoom-out, 121-149. 
Féas, E. y Steinberg, F. (2020). La politica comercial europea ante un entorno internacional cambiante. Madrid: Real Instituto Elcano.

Hamann, A., Dufour, G., Pavot, D. y Stoppioni, E. (2018). Chronique de l'OMC. Annuaire Français de Droit International, 44, 691-729.

Hauser, H. y Zimmermann, T. A. (2003). The Challenge of Reforming the WTO Dispute Settlement Understanding. Intereconomics, 38(5), 241-245. Disponible en: https://doi.org/10.1007/BF03031724.

Hillman, J. (2010). An emerging International Rule of Law? The WTO Dispute Settlement System's Role in its Evolution. Ottawa Law Review, 42 (2), 269-284.

Hillman, J. (2020). Three approaches to fixing the World Trade Organization's Appellate Body: The Good, the Bad and the Ugly. Washington: Institute of International Economic Law. Disponible en: https://bit.ly/3mqqij9.

Hoekman, B. (2016). The World Trade Order: Global Governance by Judiciary? European Journal of International Law, 27 (4), 1083-1093. Disponible en: https://doi.org/10.1093/ejil/chw063.

Hoekman, B. M. y Mavroidis, P. C. (2019a). Twin crises in the WTO, and no obvious way out. Questions of International Law, 63, 113-119.

Hoekman, B. M. y Mavroidis, P. C. (2019b). Burning Down The House? The Appellate Body in the Centre of the WTO Crisis. Florencia: European University Institute Working Papers.

Hufbauer, G. C. (2021). WTO Judicial Appointments: Bad Omen for the Trading System. Peterson institute for international economics [blog], 13-6-2011. Disponible en: https://bit.ly/3DiD1HH.

Jackson, J. H., Davey, W. J. y Sykes, A. O. (1995). Legal Problems of International Economic Relations. Cases, Materials and text on the national and international regulation of transnational economic relations. Minnesota: Wets Publishing Co.

Kanth, D. R. (2016). AB members challenge US over reappointment of Seung Wha Chang. Third World Network [blog], 24-5-2016. Disponible en: https://bit. ly/3uEc91C.

Kuijper, P. J. (2017). The US Attack on the WTO Appellate Body. Amsterdam: Amsterdam Center for International Law.

Liñán Nogueras, D. J. (2018). La internacionalización del Estado de Derecho y la Unión Europea: Una traslación categorial imperfecta. En D. J. Liñán Nogueras y P. J. Martín Rodríguez (dirs.). Estado de Derecho y Unión Europea (pp. 39-68). Madrid: Tecnos.

López-Jurado Romero de la Cruz, C. (2018). El Estado de Derecho en la acción exterior de la Unión Europea en los Balcanes Occidentales. En D. J. Liñán Nogueras y P. J. Martín Rodríguez (dirs.). Estado de Derecho y Unión Europea (pp. 425-448). Madrid: Tecnos.

Magi, L. (2020). The effect of the WTO dispute settlement crisis on the development of case law on national security exceptions: A critical scenario. Questions of International Law, 69, 29-47.

Malacrida, R. y Marceau, G. (2018). The WTO Adjudicating Bodies. En R. Howse, H. Ruiz-Fabri, G. Ulfstein y M.Q. Zang (eds.). The Legitimacy of International 
Trade Courts and Tribunals, (pp. 20-69). Cambridge: Cambridge University Press. Disponible en: https://doi.org/10.1017/9781108335690.002.

Maruyama, W. H. (2021). Can the Appellate Body Be Saved? Journal of World Trade, 55 (2), 197-230.

Mavroidis, P. C. (2017). The Gang That Couldn't Shoot Straight: The Not So Magnificent Seven of the WTO Appellate Body. European Journal of International Law, 27 (4), 1107-1118. Disponible en: https://doi.org/10.1093/ejil/chw066.

McDougall, R. (2018). The Crisis in WTO Dispute Settlement: Fixing Birth Defects to Restore Balance. Journal of World Trade, 52 (6), 867-896.

Palmeter, D., Mavroidis, P. C (2004). Dispute Settlement in the World Trade Organization. Practice and Procedure. Cambridge: Cambridge University Press. Disponible en: https://doi.org/10.1017/CBO9781139177931.

Pasoyova T., Hufbauer, G. C. y Schott, J. J. (2018). The Dispute Settlement Crisis in the World Trade Organization: Causes and Cures. Washington: Peterson Institute for International Economics.

Pauwelyn, J. (2019). WTO Dispute Settlement Post 2019: What to Expect? Journal of International Economic Law, 22, 297-321. Disponible en: https://doi. org/10.1093/jiel/jgz024.

Petersmann, E.-U. (2018a). Between "Member-Driven” WTO Governance and "Constitutional Justice": Judicial Dilemmas in GATT/WTO Dispute Settlement. Journal of International Economic Law, 21, 103-122. Disponible en: https://doi.org/10.1093/jiel/jgy004.

Petersmann, E.-U. (2018b). How should EU and other WTO members react to their WTO governance and WTO Appellate Body crises? Florencia: European University Institute Working Paper. Disponible en: https://doi.org/10.2139/ ssrn.3300738.

Roldán Barbero, J. (2018). El Estado de Derecho y la acción exterior de la UE. En D. J. Liñán Nogueras y P. J. Martín Rodríguez (dirs.). Estado de Derecho y Unión Europea (pp. 231-262). Madrid: Tecnos.

Sacerdoti, G. (2019). The stalemate concerning the Appellate Body of the WTO: Any Way out? Questions of International Law, Z, 37-58.

Wei, W. (2020). The EU search for stronger enforcement rules: Assessing the Proposed Amendments to Trade Enforcement Regulation 654/2014. Journal of International Economic Law, 23 (4), 865-884. Disponible en: https://doi. org/10.1093/jiel/jgaa033.

Weiler, J. H. H. (2001). The Rule of Lawyer and the Ethos of Diplomats. Reflections on the Internal and External Legitimacy of WTO Dispute Settlement. Journal of World Trade, 35 (2), 191-207. Disponible en: https://doi. org/10.1023/A:1016604807965. 